\title{
INTERRELACIONES ENTRE LOS PRINCIPALES PARAMETROS LIMNOLOGICOS DE LA ALBUFERA DE VALENCIA
}

\author{
M. Serra (1), M. R. Miracle (1) y E. Vicente (2) \\ Departamento de Ecología (1) y Departamento de Microbiología (2). Facutad de Ciencias Biológicas. Universidad de Valencia
}

Palabras Clave: Hypertrophic lagoons, limnological relationships, principal components, Albufera of Valencia (Spain)

\author{
ABSTRACT \\ RELATIONSHIPS AMONG THE MAIN LIMNOLOGICAL PARAMETERS OF THE ALBUFERA \\ OF VALENCIA (SPAIN), AN HYPERTROFIC COASTAL LAGOON.
}

The main limnological parameters of the Albufera of Valencia have analysed during an anual cycle in the vertical profile and different hours of the day. A principal components analysis wascarriedout with all thesedata in which 31 variablel were considered. The first there principal components accounting ftir $50 \%$ of the variance, are ecologically interpretable. They correspond respectivelly to: (1) eurotrophic level, (2) salinity whose variation is associated to the use of the Albufera as a regulat or of rice farming and ( 3 ) temperature. The first component separates a compact group of variables constituted by chlorophyll $a$ carotens and particulated phosphorus concenrrations, $\mathrm{pH}$ and copepod numbers, from another formed by alkalynity, calcium and nutrients. The second components is highly correlated with also a compact group of variables sulphate, chloride, silicate, sodium, magnesium and potasium. The third one is correlated with temperature and rotifer densities separates summer conditions from the rest. Samples in the principal components space resulted ordered according the seasons of the year, so the analysis indicates that the main source of variation is rhe annual cycle.

Another principal components analysis was undertaken with the aim of making apparent the relationships among the parameters in the daily cycle; in it each parameter was treated as a different variable for each sarnplingdate. The first principal component of this analysis is associated with the photosynthetic activity and it is rather related todepth. The second component separates midday conditions from the rest of the hours. With this analysis it is made again apparent a close association of $\mathrm{pH}$, percentage saturation oxygen, redox potencial and pigrnents index as contrasted with another laxer group of parameters formed by calcium and nutrients.

\section{INTRODUCCION}

Durante los años 1980-1981 se ha llevado a caboun estudio de los ciclos anual y diario en la Albufera de Valencia, midiéndose los parámetros limnológicos más importantes. Estos parámetros incluyen tanto variables físico-químicas como aquellas variables biológicas que, de algún modo, pueden resumir el estado de la comunidad planctónica en el lago. Algunas consideraciones subrayan el particular interés de éstos datos para explorar la estructura de la variación e interrelaciones de los principales factores o elementos Limnética 1: 9.19(1984)

(C) Asociación Española de Limnología, Madrid. Spain del sistema acuático, basándose en las especiales características de la Albufera de Valencia, que por lo demás, están descritas en otros dos artículos de este mismo volumen (Miracle et al., 1984 y Oltra y Miracle, 1984). En primer lugar, la Albufera es un sistema hipertrófico, sometido a un stress que conlleva amplias fluctuaciones de los diversos parámetros, sufriendo además un ritmo de eutrofización lo suficientemente intenso para que se pueda plasmar en cambios no sólo cíclicos sino también tendenciales en un periodo de tiempo de poco más de un año. Porotra parte, existe una manipulación de la renovación del 
agua ligada al cultivo del arroz, que se lleva a cabo con una regularidad anual. Esta manipulación tiene como consecuencia, no sólo variaciones en el nivel de las aguas, sino la exageración de las condiciones hidrológicas de la laguna,ya que se pasa de un estado de prácticamente nula renovación de las aguas a un flujo muy intenso con el consiguiente descenso de la salinidad y densidad poblacional.

El objeto de este trabajo es intentar, con ayuda de las técnicas de la estadística multivariada, poner de manifiesto y ordenar las relaciones entre los diversos parámetros limnológicos estudiados para, de este modo, establecer un modelo de las variaciones conjuntas y de las distintas tendencias de los principales elementos constituyentes de un sistema acuático hipertrófico.

\section{MATERIAL Y METODOS}

Se siguió la evolución de los principales parárnetros limnológicos en una estación de muestreo situada en el punto más profundo $(3.25 \mathrm{~m})$ de la Albufera de Valencia, situada entre "La Mata del Fang" y la orilla Este del lago. Las muestras se tomaron a 0, 1, 2 y $3 \mathrm{~m}$ de profundidad, en tres momentos distribuidos a lo largo del día. Los muestreos se efectuaron desde finales de Abril de 1980 a Julio de 1981, con periodicidad mensual en primavera y verano y cada 2 ó 3 meses durante el resto del año. Las determinaciones físicoquímicas se han llevado a cabo según las técnicas propuestos por Golterman et al., 1978. Entre los parámetros biológicos considerados, la medida de la concentración de pigmentos fotosintéticos se llevó a cabo según Strickland y Parsons, 1978; la producción primaria y asimilación obscura del plancton (método de la fijación del C14), siguiendo las indicaciones de Vollenweider, 1974; los recuentos de zooplancton según Miracle, 1974; y las estimas de los grupos bacterianos (recuento total en placa sobre medio CPS y número más problables de microorganismos desnitrificantes recontados en $D P S$ ) según se indica en Guerrero et al. 1975.

Los valores de todas las variables, con excepción del $\mathrm{pH}$ y del índice pigmentario de Margalef (D430/D665), fueron transformados previamente al cálculo de la matriz de correlación con objeto de aproximar su distribución a la distribución normal. Las variables que expresan porcentajes se transformaron tomando la raiz cuadrada positiva de sus valo- res, mientras que el resto se transformó logarítmicamente (para obviar el problema de valores 0 se sumó la unidad a los valores originales de estas variables salvo en el caso del potencial redox al cual se le sumó 69). Se hicieron dos análisis de componentes prinpales de la matriz de correlación lineal; el primero de ellos sobre el total de muestras y todos los parámetros medidos como variables ( 103 casos y 31 variables) y el segundo con las muestras agrupadas según profundidad y hora, y considerando los parámetros para cada día de muestreo como variables diferentes e independientes ( 9 casos y 76 variables). En este segundo análisis sólo se consideraron aquellos parámetros que variaban según la profundidad y el ciclo diario (señalados con un asterisco en la Tabla 1). Los análisis se realizaron en el Centro de Cálculo de la Universidad Politécnica de Valencia utilizando los paquetes estadísticos SPSS y BMDP.

\section{RESULTADOS Y DISCUSION}

En la tabla I se muestran los parámetros estudiados y sus valores medios, valores que permiten una primera aproximación a las características de la Albufera de Valencia. Se trata de aguas oligohalinas, cuyas proporciones iónicas en equivalentes disueltos corresponden a unas características mucho más cercanas a las de las aguas dulces que a las del mar. Así pues, la concentración de cloruros es siempre inferior a la de sulfatos en 1 a 3 meq y el C++ está en proporciones que pueden igualar en algunas ocasiones al $\mathrm{Na}+$. Las concentraciones de $\mathrm{Ca}++$ y $\mathrm{Mg}++$ son normalmente muy similares, si bien el $\mathrm{Ca}++$ muestra en la mayoría de los casos valores ligeramente superiores. Las aguas de la Albufera corresponden a las relaciones siguientes: $\mathrm{SO}_{4}>\mathrm{Cl} \longrightarrow$ Alc y $\mathrm{Na}+2 \mathrm{Ca}++2 \mathrm{Mg}++>\mathrm{K}+$.

Por otra parte, los valores encontrados para los demás parámetros son consecuencia de la elevada producción primaria de la laguna. Así pues, presenta: (1) una reserva alcalina muy baja, (2) un $\mathrm{pH}$ extraordinariamente alto con un marcado ciclo diario y anual; y (3) niveles de oxígeno fluctuantes, pero normalmente de sobresaturación, si bien el potencial redox se mantiene siempre bajo como consecuencia de la gran cantidad de compuestos orgánicos presentes en el agua. Además, todos los parámetros biológicos ponen de manifiesto el estado de extrema hipertrofia de la laguna.

La variación anual de algunos parámetros viene 
Tabla I - Medias, desviaciones típicas y número de observaciones de las variables estudiadas Junto al nombre de la variable se indica la abreviatura utilizada en las figuras Las variables señaladas con * son las incluidas en el análisis de componentes principales II Means, standard desviations and number of observations of the parameters studied. Abbreviations used in the figures are indicated before its complete name Parameters marked with * are those in which principal components analysis 11 is based.

VARIABLE

Temperatura. Temp. ( $\left.\mathrm{C}^{\mathrm{Q}}\right)$

Conductividad, Cond. ( $\mu \mathrm{Si} / \mathrm{cm}$ )

* pH

* Alcalinidad, Alc (meq/l)

*Potencial Redox, Eh ( $\mathrm{mV}$ )

Sulfatos, $\mathrm{SO}^{2-}$ (meq/1)

*Nitracos, $\mathrm{NO}_{3}^{*}(\mu \mathrm{mol} / 1)$

Nicritos, $\mathrm{NO}_{2}^{-}(\mu \mathrm{mol} / 1)$

*lón Amonio, $\mathrm{NH}_{i}^{+}(\boldsymbol{\mu m o l} / 1)$

Silicatos, $\mathrm{SiO}_{3}=(\mu \mathrm{mol} / \mathrm{l})$

Cloruros, $\mathrm{Cl}(\mathrm{meq} / \mathrm{I})$

Sulfuros, $S-(\mu \mathrm{g} S / 1)$

Ión Sodio, $\mathrm{Na}+(\mathrm{meq} / 1)$

Ión Potasio $\mathrm{K}+(\mathrm{meq} / \mathrm{l})$

*Ión Calcio, $\mathrm{Ca}^{2}+(\mathrm{meq} / \mathrm{l})$

Ión Magnesio, $\mathrm{Mg}^{2-}(\mathrm{meq} / \mathrm{l})$

Oxígeno, $\mathrm{O},(\mathrm{mg} / \mathrm{l})$

* \% Saturación $\mathrm{O}_{2}, \% \mathrm{~S}_{2} \mathrm{O}_{2}(\%)$

*Fosfato Soluble, $\mathrm{PO}_{4}{ }^{3-}(\mu \mathrm{mol} / \mathrm{l})$

Fosfato Particulado, PP $(\mu \mathrm{mol} / \mathrm{l})$

Clorofila $a, \mathrm{Cl}$ u $(\mu \mathrm{g} / \mathrm{I})$

Carotenos, Car ( $\mu \mathrm{g} / \mathrm{l})$

*Indice Pigmentos: D430/D665 (I.P)

Producicin Primaria. Pl ( $\mathrm{mg} \mathrm{C} / \mathrm{m}^{3} \cdot \mathrm{h}$ )

Asimilacion Oscurat. AS O ( $\left.\mathrm{mg} \mathrm{C} / \mathrm{m}^{3} \cdot \mathrm{h}\right)$

Dox Producción Primanta. Do Pl (mg $\left.\mathrm{C} / \mathrm{m}^{3} \cdot \mathrm{h}\right)$

Dox Asimilación Oscura. Dor As O ( $\mathrm{mg} \mathrm{C} / \mathrm{m}^{3} \cdot \mathrm{h}$ )

No Microurganismos Torales. M T coulonias ml)

$\mathrm{N}^{\mathrm{O}}$ Microorganismos Desnitrificanies, MDN (NMP/100ml)

$\mathrm{N}^{\mathrm{e}}$ de Rotíferos, RF (Ind./1)

$\mathrm{N}^{*}$ de Copépodos y Cladóceros, CC (Ind/1)

\section{DESVIACION} TIPICA

$6.00 \quad 103$

232.68

0.77

0.52

258.45

0.83

23.81

2.55

15.15

68.02

1.05

0.07

1.21

0.04

0.54

0.42

4.46

41.82

$0.2^{\circ}$

4.81

198.19

67.42

1.03

236.33

16.34

34.62

16.72

130312.52

3644.91

2534.29

187.29
Ne CASOS

91

103

103
70

70

90

103

90

78

78

78

103

100

87

87

87

103

103

103

103

103

103

103

90

90

78

78

103

87

97

97 ilustrada en la figura I. La gráfica correspondiente a la temperatura pone de manifiesto unas aguas cálidas con una estación fría muy suave, con temperaturas no inferiores a $\operatorname{los} 10^{\circ} \mathrm{C}$. Los otros tres parámetros representados, están ligados a la variación de la actividad fotosintérica con un elevado máximoen primavera y un mínimo en otoño-invierno. Así, la clorofila $a$ presenta una evolución paralela a la del $\mathrm{pH}$ e inversa a la de la alcalinidad. Sin embargo, la clorofila a muestra una variación algo más compleja que el $\mathrm{pH}$, ya que a la floración primaveral le sigue un mínimo relativo, con una posterior recuperación en el mes de agosto. La oscilación descrita puede interpretarse como originada por un agotamiento de los nutrientes, consumidos en el crecimiento primaveral. En cambio, la variación del $\mathrm{pH}$ promedio está enmascarada por las importantes diferencias que se presentan en verano para las distintas profundidades y horas del día, como queda reflejado en los mayores valores de la desviación típica representada en la figura 1. Esto es debido a que la menor rurbulencia y elevada temperatura del agua provoca un importante gradiente espacial y temporal del equilibrio carbónico-carbonatos, que depende principalmente de las diferencias entre las actividades respiratorias y forosintéticas. Este efecto no aparece en la representación de la alcalinidad puesto que los valores utilizados para este parámetro son los totales integrados para toda la columna de agua por lo que la variación espacial nose considera. Además, puesto que en la Albufera la 

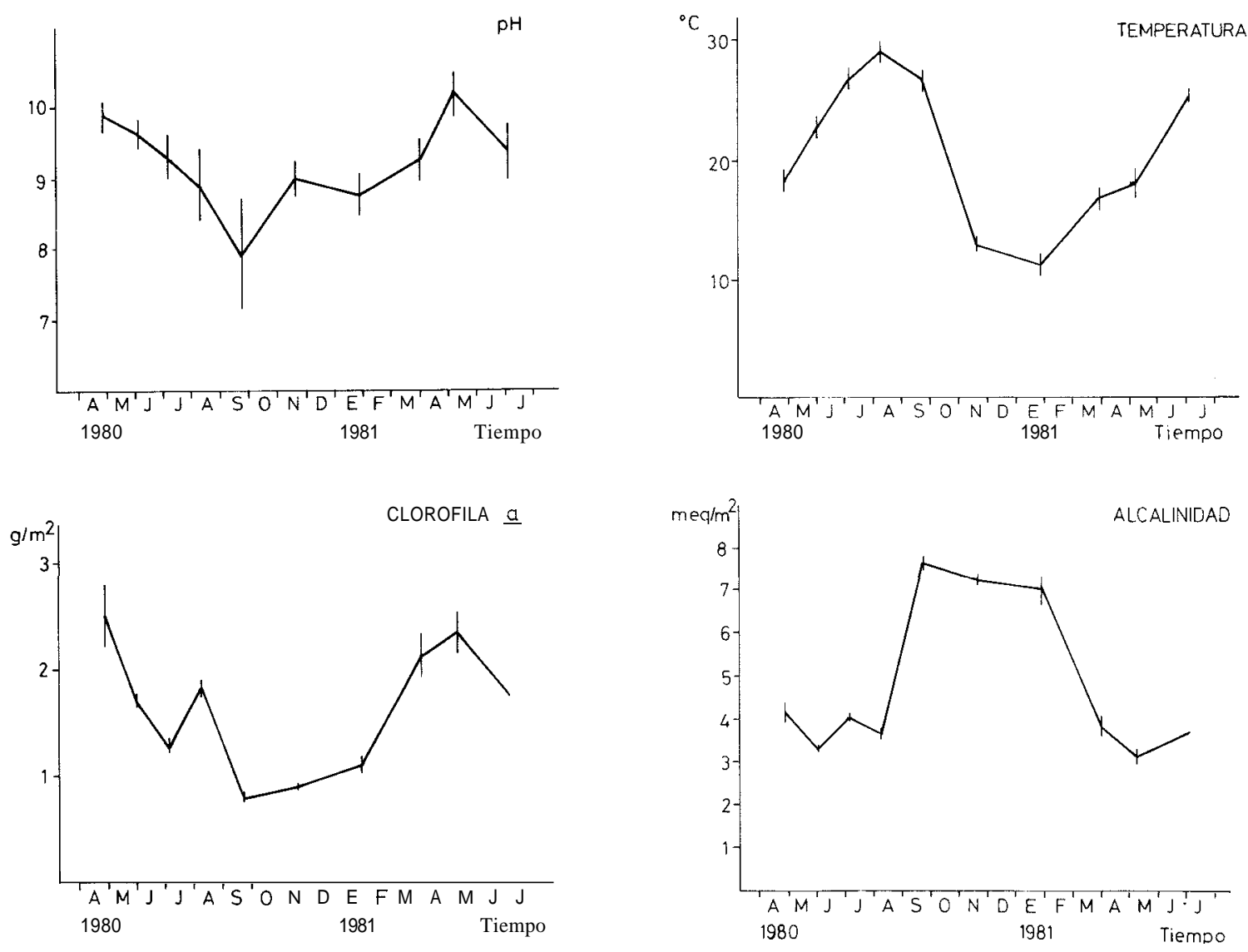

I Igura ] - Variación estacional del pH, temperarura, clorofila a y alcalinidad. La linea de trazo gruesa une los valores medios mensuales de cada muestra puntual correspondiente a las diverasas horas del día y profundidades en el caso del pH y de la temperatura, y de las integraciones por columna de agua en el caso de la clorofila a y de la alcalinidad. Los segmentos verticales (trazo fino) centrados en las medias corresponden a, más y menos, las desviaciones tupıcas de los valores de cada mes.

Seasonal variation of $\mathrm{pH}$ temperature, chlorophyll $a$ and alkalinity. The thick line unites the monthly means over all punctual samples (corresponding to various depths and hours of the day) for temperature and $\mathrm{pH}$ and over the integrations per column of water for chlorophyll u and alkalinity. Thin vertical line represent plus and minus one standard deviations for each month.

reserva alcalina es baja, las variaciones del pH son mucho más agudas que las de aquel parámetro.

La ordenación de las correlaciones entre los diferentes pares de variables (Fig. 2) revela la existencia de cuatro conjuntos de parámetros estrechamente asociados constituidos por: (1) los iones mayoritarios que determinan la salinidad de las aguas; (2) los parámetros biológicos (pigmentos fotosintéticos, crustáceos, fósforo particulado) y los físico-químicos asociados directamente a la actividad fotosintética como el pH y la concentación de oxígeno; (3) los nutrientes, alcalinidad y calcio, que varian inversamente a los del grupo anterior, mostrando fuertes correlaciones negativas con aquellos y (4) un grupo de variables que revelan la existencia de condiciones reductoras en el medio $(\mathrm{NH}+4$, potencial redox, sulfhídrico, excreción de material orgánico soluble)

\section{ANALISIS DE COMPONENTES SOBRE LA TOTALIDAD DE LOS DATOS (ACP I)}

Con el objeto de explorar más profundamenteestos datos se llevó a cabo un análisis con las 103 muestras, tomadas a distintas profundidades y horas del día durante un ciclo anual, consideradas independientemente y caracterizadas por las 31 variables de la tabla 1 . Los resultados de este análisis son ecológicamente interpretables, acumulando las tres primeras componentes principales una explicación del $50 \%$ de la varianza (Tabla 2).

En la figura 3 se muestran las correlaciones entre los parámetros limnológicos y las tres primeras 


\section{CODIGOS DE REPRESENTACION}
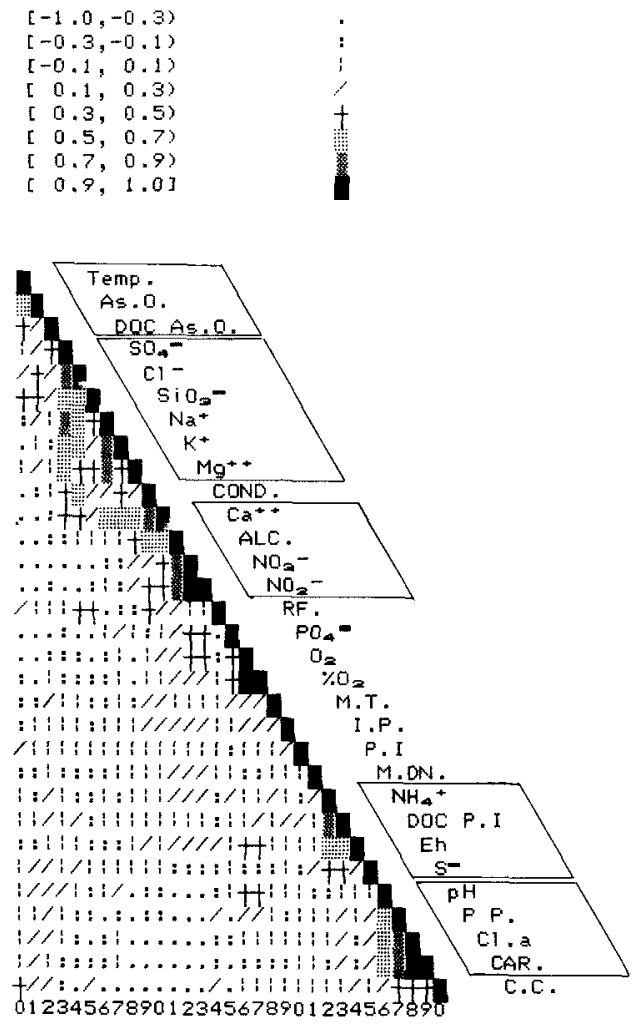

Figura 2.- Matriz de correlación entre las diversas variables urdenadas según un análisis de agrupación. Se recuadran los grupos de variables más asociadas entre sí - Correlation matrix between the studied variables. They are ordered according to a cluster analysis. Groups of closely associated variables are indicared

componentes. En el primer eje se separan las variables relacionadas con el crecimiento algal (clorofila a y carotenos) y la biomasa total (fósforo particulado, número de artrópodos) de aquellos que representan nutrientes (fósforo soluble, nitratos y nitritos) puesto que a los explosivos crecimientos algales va asociado un consumo de nutrientes. Así pues, el primer eje se interpreta como una ordenación de los distintos estados de eutrofia, o de actividad fotosintética, por los que pasa el lago. El pH, por su localización, está asociado al primer grupo de variables, mientras que la alcalinidad y la concentración de iones $\mathrm{Ca}++$ se agrupan junto con los nutrientes. Ello indica que la tendencia a pH elevados de estas aguas se debe a la alta
Tabla II.- Varianza explicada (valores propios) y porcentaje acumulado de la varianza total para las tres primeras componentes resultantes de los análisis de componentes principales I y II.

Common variance (eigenvalues) and cumulative percentage of variance accounted for respectively the first three components of principal components analyses $I$ and II

$\begin{array}{ccc} & \begin{array}{c}\text { VARIANZA } \\ \text { EXPLICADA }\end{array} & \begin{array}{c}\% \text { ACUMULADO DE } \\ \text { VARIANZA }\end{array} \\ \text { ANALISIS DE COM- } & & \\ \text { PONENTES PRINCI- } & & \\ \text { PALES I } & & 21.9 \\ \text { Componente I } & 6.781 & 39.6 \\ \text { Componente II } & 5.498 & 50.0 \\ \text { Componente III } & 3.218 & \\ \text { ANALISIS DE COM- } & & \\ \text { PONENTES PRIN- } & & \\ \text { CIPALES II } & & 32.8 \\ \text { Componente I } & 24.943 & 50.3 \\ \text { Componente II } & 13.245 & \end{array}$

actividad fotosintética, lo que provoca la precipitación de carbonatos y la disminución de la reserva alcalina. $\mathrm{La}$ asociación entre $\mathrm{Ca}++$ y alcalinidad, que se pone de manifiesto tanto mediante el ACP I como en el valor de su correlación ( $\mathrm{r}=0.66$; fig. 2 ), señala que la precipitación de carbonatos ocurre en gran parte en forma de $\mathrm{CO}_{3} \mathrm{Ca}$. La posición que ocupa el fóforo soluble en la primera componente se puede explicar por dos causas en relación al crecimiento algal: la precipitación como hidroxiapatitos del fósforo a $\mathrm{pH}$ altos y su incorporación al citoplasma celular. Esta última explicación se hace extensiva a los nitratos.

El segundo eje está correlacionado positivamente con los iones que determinan la salinidad de las aguas $\left(\mathrm{Cl}^{-}, \mathrm{SO}_{4}^{2}-\mathrm{SiO}_{3}{ }^{2}, \mathrm{~K}+\right.$ y $\left.\mathrm{Ca}++\right)$ y por tanto asociado al estado de mineralización de las mismas. La concentración de 02 y el potencial redox, presentan correlaciones negativas con este segundo eje. La figura 4 ayuda a interpretar estos resultados ya que muestra la dependencia entre la mineralización de las aguas y el estado de apertura o cierre de las compuertas. Teniendo en cuenta que el flujo de agua que se impide con las compuertas cerradas es siempre en dirección al mar, las compuertas cerradas provocan un aumento de la salinización ya que evitan el lavado del agua que, por otra parte, debido a la evaporación y disolución del sedimento va sufriendo una mineralización. Todo ello está favorecido por las características de la laguna: aguas someras y calidas, con algunos aportes subterráneos por filtración.

La estrecha relación entre este conjunto de iones agrupados por el ACP I, ya es aparente en el 


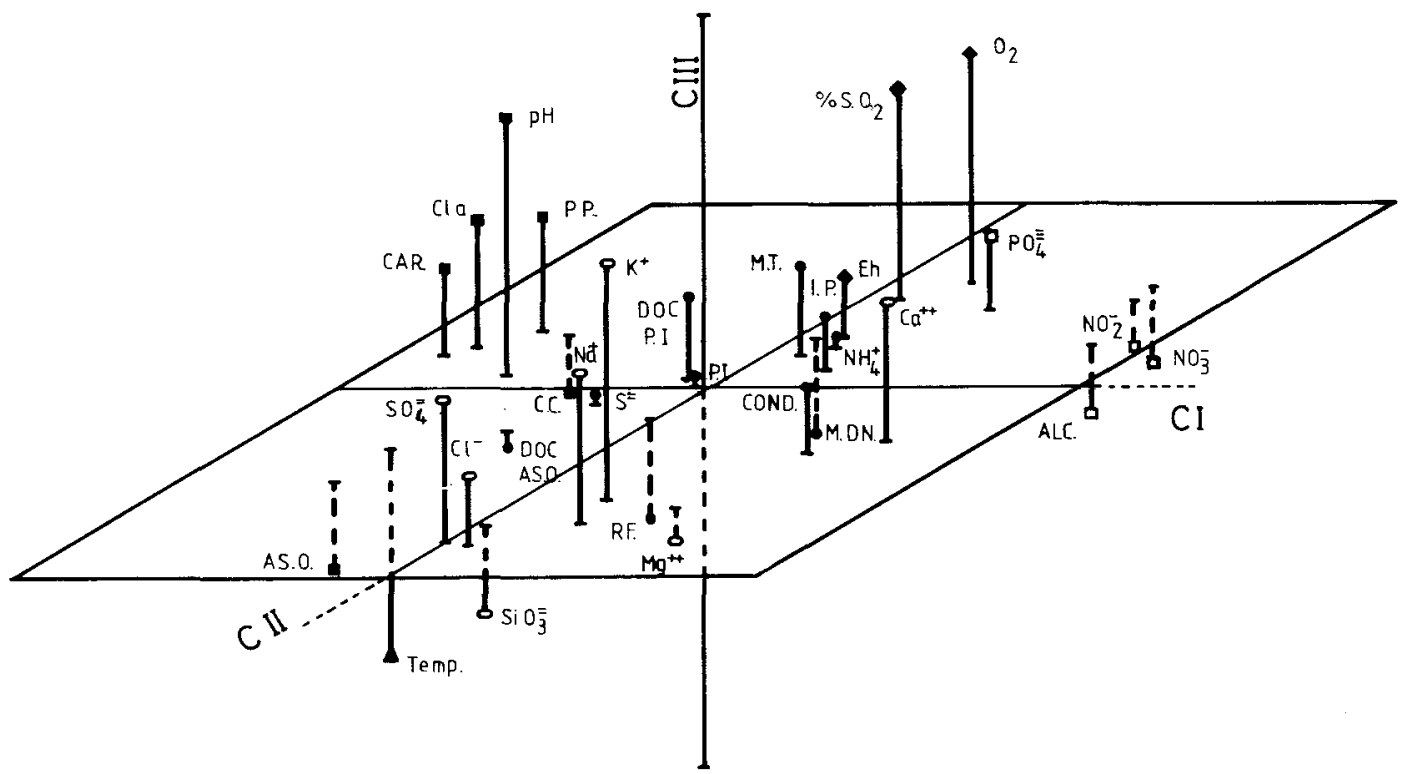

Figura 3.- Posición relativa de los distintos parámetros en el espacio de las tres primeras componentes principales resultantes del a nálisis con todos los datos (ACPI). Las coordenadas son los repectivos coeficientes de correlación. Variables asociadas a: a la actividad fotosintética, o 1 a salinidad, el oxígeno y el potencial redox, $\square$ los nutrienres, m correlaciones bajas. Relative position of each parameter in the space dimensioned by the first three principal cornponents from the analysis with all data and samples considered indepently. The coordinares are the respective loadings or correlation coefficients. Variables associated to: photosynthetic activity o salinity. oxygen and redox potential. $\square$ nutrients, low correlations

análisis de las correlaciones dos a dos entre parámetros (Fig. 2). Ligado a los habituales parámetros de proporcionalidad constante aparece el $\mathrm{SiO}_{3}^{2}$; demostrándose así la relativa poca importancia que en la Albufera poseían en el periodo de estudio las poblaciones de diatomeas, como cabe esperar en unas aguas muy eutróficas. Además, es general en estas lagunas litorales valores altos de silicio en verano (López, 1983), cuya explicación podría ser el descenso importante de oxígeno en el fondo, que facilita la redisolución del silicato del sedimento,y la elevada temperatura que incrementa la solubilidad del mismo, junto con la infiltración de posibles aportes subterráneos que son ricos en silicio.

La oposición, dentro de la segunda componente, entre las especies químicas asociadas a la mineralización y las variables relacionadas con el oxígeno, aparece comunmente en los análisis de componentes principales sobre parámetros limnológicos medidos en varias épocas del año (López, 1983; Estrada, 1975). Si bien se podría aducir que es debida a algunas relaciones de tipo químico, en realidad va ligada al ciclo anual de periodos de mezcla y reposo de las aguas. Así, en la Albufera esta oposición vendría dada, en primer lugar, por la coincidencia en los meses de verano (Julio y Agosto) de una elevada mineralización -determinada por el régimen de compuertas y por las altas temperaturas- con una acumulación de materia orgánica -determinada por el crecimiento fitoplanctónico- y, en consecuencia, con un incremento del consumo de oxígeno y del carácter reductor del agua. En segundo lugar, el flujo que disminuye la fracción mineral del agua por lavado cuando se abren las compuertas, afectará de una forma similar la densidad de población de los organismos y la cantidad de materia orgánica del agua. Esto, unido a una mayor turbulencia que deshace los gradientes y a la disminución de la temperatura, ocasiona el correspondiente aumenta de la concentración de oxígeno y del potencial redox en toda la columna de agua.

El tercer eje separa las condiciones particulares de los meses de verano para contraponerlas sobre todo a las de primavera. Este eje está en correlación negativa con la temperatura $y$, en menor medida, con el número de rotíferos y el silicato; se correlaciona a su vez positivamente con el $\mathrm{pH}$ y la concentración y el porcentaje de saturación del oxígeno.

Las tres componentes que se han tenido en cuenta asocian las muestras atendiendo, sobre todo, a la época del año en la cual fueron tomadas. Las muestras se 


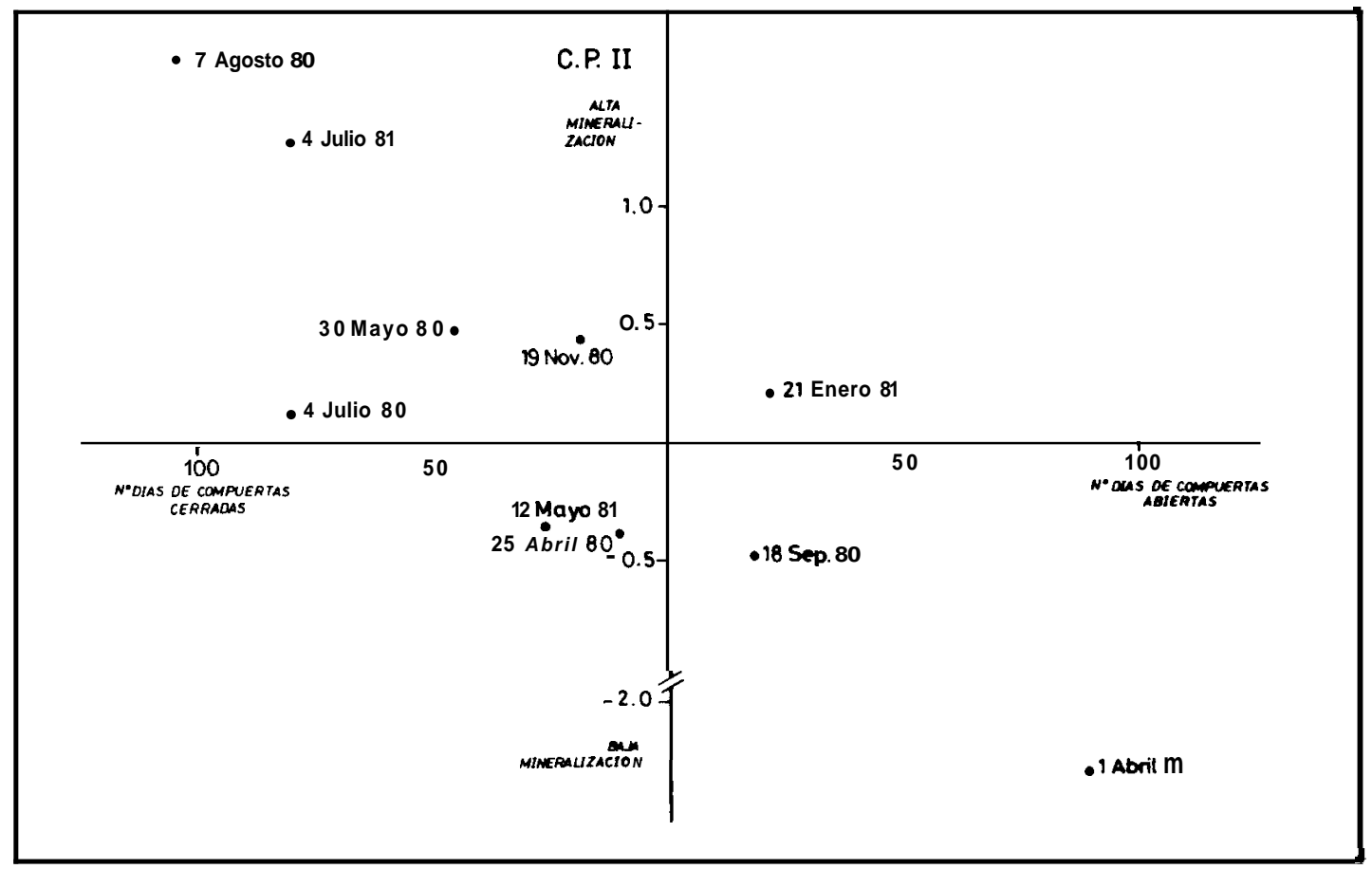

Figura + - Relación rntre la componente principal II (ACP I) asociada a la mineralización (ordenadas) y el régimen de desagüe del lago (abcisas). Las distintas fechas de muestreo vienen determinadas por el número de días transcurridos después del cierre o apertura de las compuertas y por el valor medio de aquella componente. - Relationships between the principal component II (ACP I) associated with mineralization (ordinates) and rhe manipularion of water flow in the Albufera (abscissas). The sampling dates are determined by the number of days following the closing or the opening of the lock-gates and the mean of the factor scores of that component.

agrupan en cuatro grandes conjuntos en el espacio definido por las tres primeras componentes (Fig. 5) que corresponden a las distintas épocas del año. Estas tres primeras componentes difícilmente dejan entrever alguna ordenación de las muestras que obedezca a la hora del día o profundidad de su procedencia, puesto que el ciclo anual es la principal fuente de variación común de los datos. La variación diaria se esconde en componentes de poca significación cuyos factores de carga son sólo importantes para un número muy reducido de variables. Por ejemplo, tal es el caso de la séptima componente principal que explica el $2 \not \%$ de la variación total y que está determinada exclusivamente por la producción primaria. Esta producción es sólo importante en superficie a las horas de luz.

Análisis de componentes principales para el estudio del ciclo diario (ACP II)

Con el objeto de poner de manifiesto las interrelaciones entre variables debidas al ciclo diario, se llevó a cabo un segundo análisis de componentes principales (ACP II). En primer lugar, se realizó una selección de parámetros, deshechandose aquellos que no presentaban una oscilación importante en razón de la hora o de la profundidad. Tampocose ha considerado la producción primaria. En el análisis en cuestión, un único parámetro se subdivide en distintas variables atendiendo al mes de muestreo, de modo que cada parámetro está tratado como varias variables en número igual al de meses en que ha sido medido (p.e., el $\mathrm{Ca}++$ del mes de Agosto y el $\mathrm{Ca}++$ del mes de Septiembre son variables distintas). Tal reestructuración de datos lleva consigo una drástica reducción del número de casos, que se limitan a nueve y son definidos por la combinación de la profundidad $(\mathrm{O}, 1$ y $3 \mathrm{~m}$ ) y la hora (amanecer, mediodía y noche) de las muestras.

Este segundo análisis explica, mediante las dos primeras componentes principales, el 50\% de la varianza total (Tabla 2). La posición que ocupan los casos en el espacio que generan las dos primeras com- 


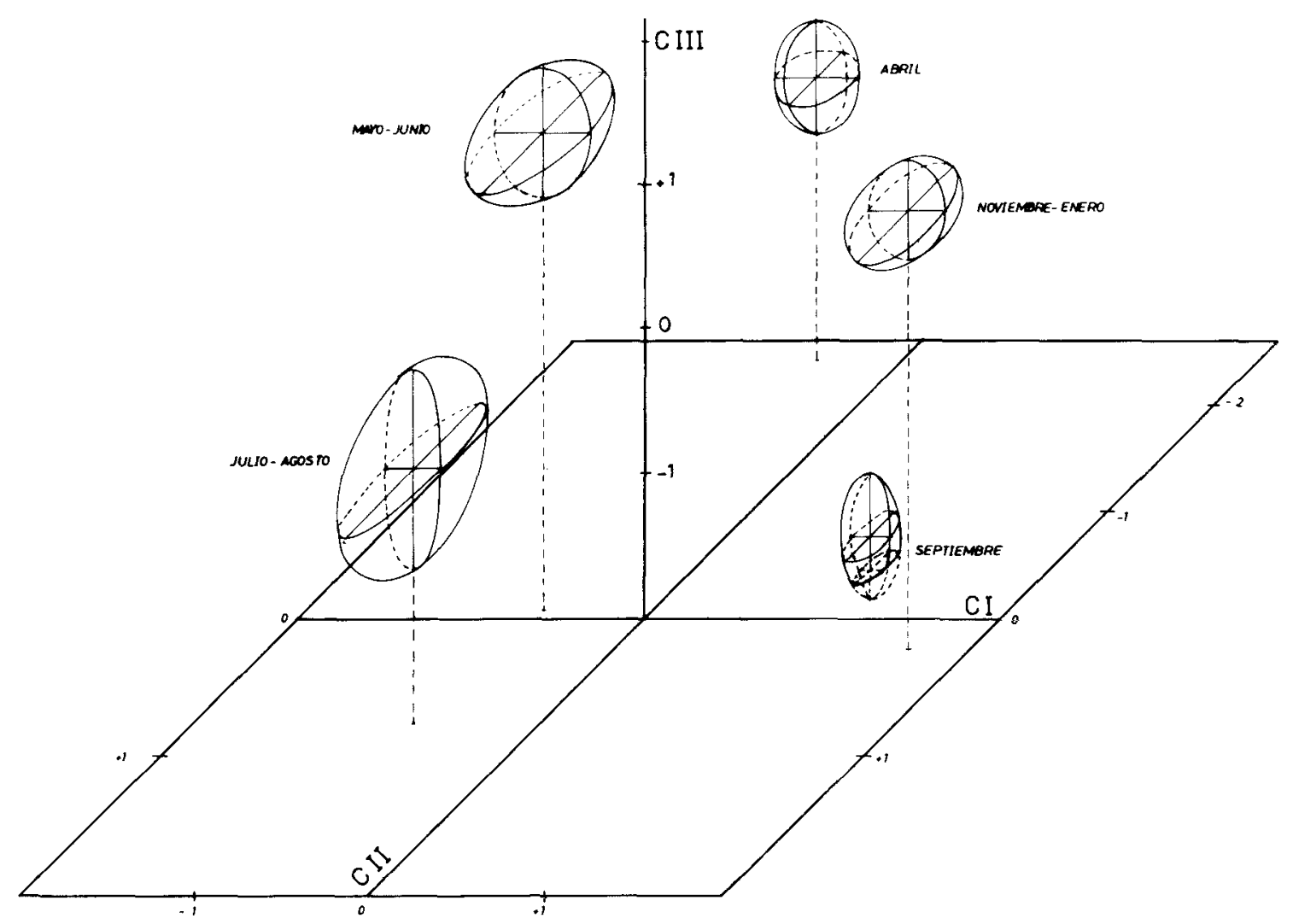

Figura 5 - Agrupación de las diferentes muestras o casos segun los meses del año en el espacio dimensionado por las tres primeras componentes del ACP I Ios elipsoides están centrados por los valores medios de las componentes para las muestras correspondientes a los meses indicados, siendo la longitud de sus semejes igual al valor de las desviaciones típicas - Clustering of rhe different samples or cases according to the ames of the year an the space dimensionated by the frist threecomponents from ACP I The elipsoids are centead by the mean tactor soures of rlie components for the simples corresponding to rhe indicated months being the sember length equal to the standard deviation values

ponentes se puede observar en la figura 0 Aquí las muestras se segregan, tanto por la hora corno por la profundidad de la toma. Las muestras de distintas profundidades tomadas al amanecer distan menos entre ellas de lo que lo hacen las tomadas a otras horas del día. Ello se explica por las condiciones de mezcla que dominan en el lago, por lo demás muy somero, de forma qiie los gradientes que se llegan a establecer respecto a la profundidad tienen un carácter transitorio (se establecen durante el dia y se anulan a lo largo de la noche).

En la figura 7 se han representadolascorrelaciones entre las variables usadas en el análisis y las dos primeras componentes, En ella se distingue un grupo de parámetros (porcentajede saturación de (2), Eh y $\mathrm{pH}$ ) que alteran poco su correlación con el primer eje a lo largo del año. También destaca la semejanza entre las posiciones ocupadas por el $\mathrm{pH}$ y el porcentaje de saturación de 0. 1.a componente I debe interpretarse corno representante de la actividad fotosintética y de las condiciones oxidantes, versus condiciones más reductoras,y está ligada en mayor grado a la profundidad. Diferencia especialmente las condiciones en la capa de agua del fondo y en todo el perfil al amanecer frente a las de la superficie a las demás horas. Por ello $\mathrm{pH}$, Eh y porcentaje de saturación de $0_{2}$ tienen una $\mathrm{po}^{-}$ sición bien definida, con correlaciones positivas con la componente, durante todo el año; más o menos asociado está también el índice de pigmentos, si bien en épocas de crecimiento masivo éste no presenta ya $\mathrm{l}_{a}$ misma pauta de variación en profundidad. Otros parámetros, como los iones salinos y compuestos reducidos 


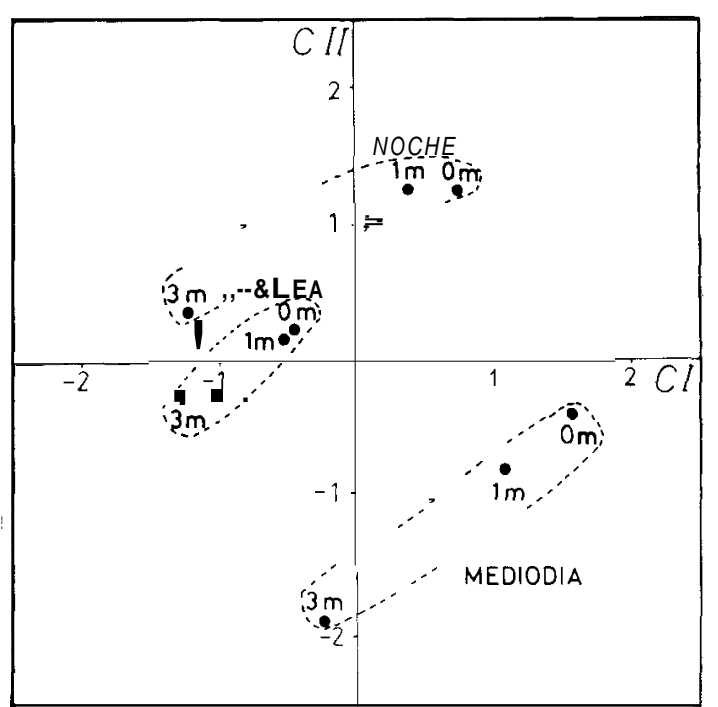

Figura (- Ordenación de discintos periodes del diay profundidade en el espacio definido por las dos primeras componentes principales resultantes del ACP II. Las profundidades ( 0,1 y 3 metros) muestreadas dentro de un mismo periodo del día se engloban con una linea de trazos. - Ordination of different periods of the day and depths in the space dimensioned by the frist two principal components from the ACP II. Depths $(0,1$ and 3 meters) sampled within the same period of the day are enclosed by dash line

presentan correlaciones negativas puesto que sus concentraciones máximas se dan en las aguas del fondo; esto ocurre,por ejemplo, con el $\mathrm{Ca}++\mathrm{y} \mathrm{NH} 4+$ (Fig. 7) los cuales, sin embargo, presentan una variación anual bastante grande haciéndose su correlación menos negativa en verano. Esto puede ser debido a que el $\mathrm{Ca}++$ es mucho menos soluble cuando la temperatura es elevada; tal circustancia hace que el calcio disminuya en toda la columna de agua en verano no observandose un incremento en el fondo. Igualmente, el amonio también disminuye en verano,como pauta general de los nutrientes, presentando fuertes disminuciones en profundidad debido a la nitrificación. La segunda componente separa sobre todo las condiciones que se dan al mediodía de todas las demás, presentando todos los parámetros grandes diferencias según la época del año. En especial, los antes mencionados $\mathrm{pH}$, porcentaje de saturación con 02 y redox, resultan dispersados a lo largo de este eje, siendo los extremos más contrapuestos los correspondientes a los meses de floración algal de primavera con respecto a los correspondientes en verano.

\section{CONSIDERACIONES GENERALES}

El análisis de componentes principales practicado con la totalidad de datos y las muestras consideradas independientemente (ACP I) confirma que hay unas direcciones de variación común de los parámeros limnológicos muy claras y son las mismas que se ponen de manifiesto, una y otra vez,cuando estos análisis han sido utilizados sobre otros conjuntos de datos similares. La mineralización de las aguas y grado de eutrofia han resultado ser factores de variación común, en este orden de importancia, cuando se hanconsiderado datos procedentes de una serie amplia de embalses (Estada, 1975, 1978; Margalef et al. 1982) o lagunas litorales (López, 1983). Cuando se trata de un ciclo anual como el que nos ocupa, la variación más importante es la debida a lo que podríamos asimilar a la eutrofia o periodos de máximo crecimiento del plancton frente a otros de menor crecimiento. Esto se plasma en la asociación de dos grupos de variables: la clorofila, el pH, la densidad de organismos planctónicos, el fósforo particulado (o fósforo reactivo en los casos citados de las lagunas litorales y embalses) por un lado y, en contraposición, la alcalinidad y nutrientes (nitratos, amonio y fósforo soluble) por otro lado. En el caso de ciclos anuales de la densidad de las poblaciones planctónicas, también un primer factor va ligado a los periodos de mezcla y crecimiento de los organismos (Miracle, 1974). En la Albufera y debido a las fluctuaciones tan intensas de salinidad -hecho general en las lagunas litorales- durante el ciclo anual, la mineralización aparece en segundo lugar asociando los iones mayoritarios determinantes de la misma, en contraposición con el oxígeno, redox y variables asociadas. En dicha lagunas y en el estudio de una serie de lagunas litorales (López, 1983), las condiciones de verano frente a las condiciones de las demás épocas del año, también acostumbran a salir como determinantes de alguna componente principal en el análisis de ciclos anuales. Así, la temperatura es una variable con un coeficiente de carga importante y en casos en que no se incluye en el análisis puede presentar una correlación alta, calculada a posteriori, con alguna componente principal derivada del análisis (Miracle, 1974).

En el caso de la Albufera, otras variables importantes que determinan la componente principal asociada a la temperatura, son las más influenciadas por la estratificación de las aguas que tiene lugar en la época de verano como es el caso, entre otras, del $\mathrm{pH}$ y concentración de 02 por un lado y redox y alcalinidad por otro. Esta componente asociada a la estratificación de las aguas también se obtiene en los análisis realizados 


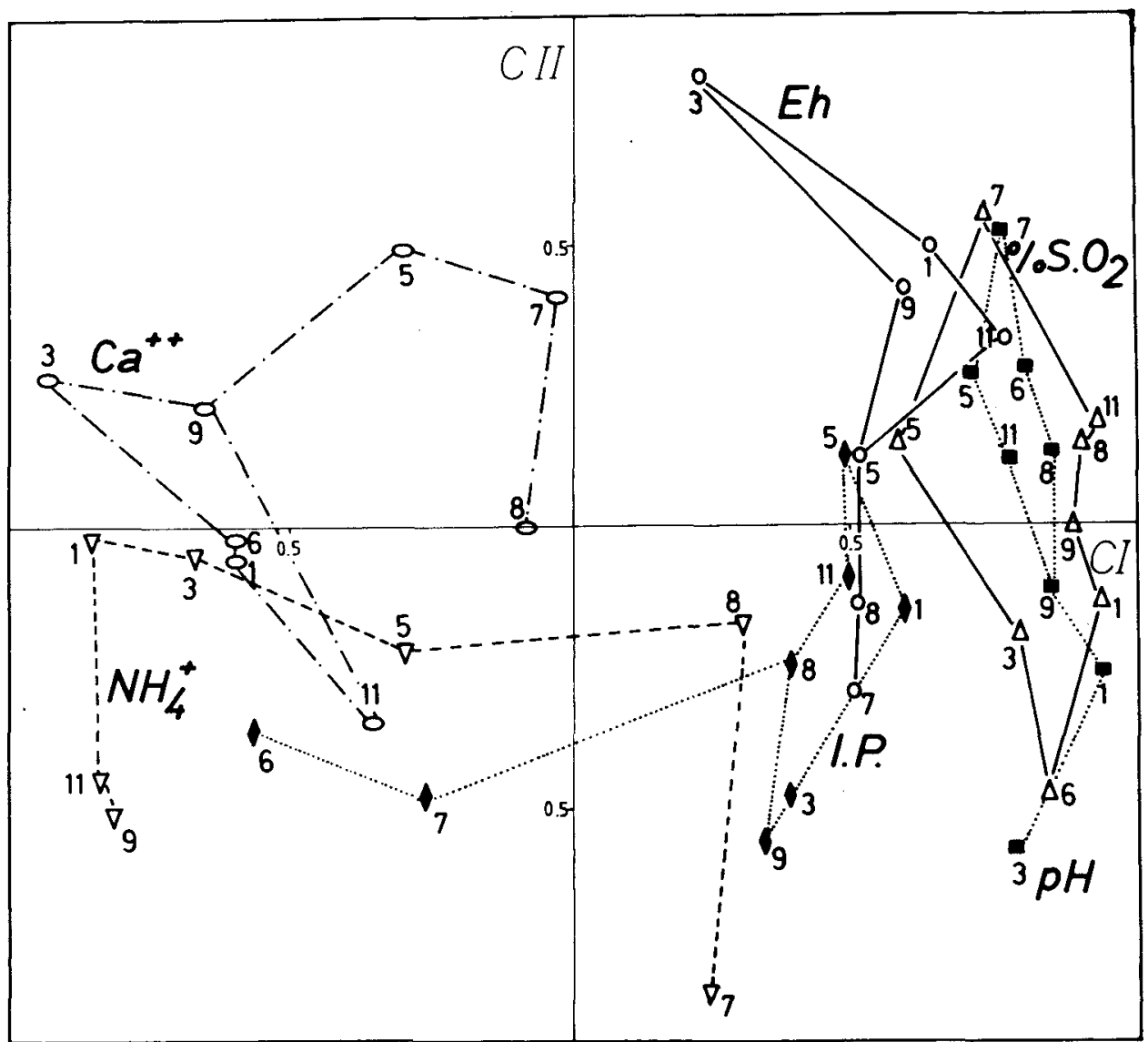

Figura 7.- Posición relativa de algunos parámetros en el espacio definido por las dos primeras componentes principales resultantes del ACP Il. Cada parhmetro se representa por una serie de puntos unidos, cuyas coordenadas son el coeficiente de correlación con los ejes en cada uno de los meses estudiados, que se indican con su ordinal correspondiente. $\mathbf{O}$ Calcio. $\nabla$ Amonio, Indice de pigmentos $\mathrm{D},,,, \mathrm{D}_{\mathrm{or} i s}, \square \mathrm{pH}, \mathrm{O}$ redox, $\Delta \%$ saruración de oxígeno). - Relarive position of several parameters in the space dimensioned by the first two principal components from ACP II. Each parameter is represented by a series of joined points, whose coordinates are the respective loading or correlation coefficients in each studied month, which are indicated by their corresponding number. $\bigcirc \mathrm{Calcium}$

$\nabla$ Amonium, Pigments index $\mathrm{D}_{i t i} \mathrm{D}_{6,5,5} \square \mathrm{pH}, \bigcirc$ redox, $\Delta \%$ saturation of oxygen.

en grupos de embalses. siendo más característica cuando se efectuan a partir de los datos de abundancia de los organismos planctónicos (Margalef et al., 1982).

La idea de buscar interrelaciones debidas al ciclo diario, nos llevó a aceptar que éstas son enormemente dependientes de la época del año, siendo en extremo difícil obtener regularidades excepto con parámetros característicos distribuidos en gradientes verticales como son porcentaje de saturación de $\mathrm{O}$, potencial redox y $\mathrm{pH}$. Conclusiones parecidas sobre la gran variación a lo largo del año de las relaciones entre parámetros, se pueden extraer de los trabajos citados sobre los embalses (Estrada, 1975 y 1778).

\section{AGRADECIMIENTOS}

Deseamos agradecer al ingeniero del ICONA R. Ruano las facilidades prestadas para la realiza- 


\section{ción de las experiencias de campo en el presente trabajo.}

\section{BIBLIOGRAFIA}

Estrada, M. 1975. Statistical considerations of some limnological parameters in Spanish lagoons. Verh. Internat. Verein. Limnol., 19: $1848-1859$

Estrada, M. 1978. Relatinships among biological and physicochemical parameters in Spanish reservoirs. Verch. Internat. Verein. Limnol., 20: 1642-1646.

Golterman, H.L., R.S. Clymo and M.A.M. Ohnstad., 1978. Methods for physical and chemical analysis of fresh waters. Blanckwell Scientific Publications. Oxford.

Guerrero, R., F. Roda., C. Albella and F. Torrella., 1975. Optimal growth temperarures and media parameters of bacterial comunities from lakes of Wifferent trophic states. Verh. Internat. Verein. Limnol., 19: 2620-2626.
López, P. 1983. Aguas salinas epicontinentales próximas a la costa mediterránea española. Estudio del medio. Tesis Doctoral. Universidad de Barcelona.

Margalef, R., M. Mir. y M. Estrada. 1982. Phytoplankton composition and distribution as an expression of properties of reservoris. Can. Water Res. J., 7: 26-49.

Miracle, M.R., 1974. Estructura y dinámica de las poblaciones de la comunidad zooplantónica del lago Banyoles. Tesis Doctoral Universidad de Barcelona.

Miracle, M.R., P. García y E. Vicente., 1984. Heterogeneidad espacial de las comunidades fitoplantónicas en la Albufera de Valencia. Limnetica 1: 20-31

Oltra, R. y M.R. Miracle. 1984. Comunidades zooplantónicas de la Albufera de Valencia. L.mnetica 1: 51-61

Strickland, J.D.H. and T.R. Parsons., 1978. A practica1 handbook of sea water analysis. Bull. Fish. Res. Bd. Can., 167: 1-203.

Vollenweider, R.A., 1974. A manual on methods for measuring primary production in aquatic enviroments. Blackwell Scientific Publications Oxford. 